\title{
Atributos físicos e químicos de um Latossolo Amarelo distrófico coeso e crescimento radicular de Brachiaria decumbens submetido à subsolagem e fertilização
}

\author{
Erivaldo de Jesus da Silva', Petterson Costa Conceição Silva², Fábio Farias Amorim ${ }^{3}$ \\ Ralph Bruno França Brito', Bruce Mota Pamponet', Joelito de Oliveira Rezende' \\ Universidade Federal do Recôncavo da Bahia, Cruz das Almas, BA, Brasil \\ Universidade Federal do Ceará, Fortaleza, CE, Brasil \\ Universidade Federal do Rio Grande do Sul, Porto Alegre, RS, Brasi \\ *Autor correspondente, e-mail: pitt2mil@gmail.com
}

\begin{abstract}
Resumo
Degradação de pastagem é um processo evolutivo de perda de vigor, produtividade e capacidade de recuperação natural das pastagens, inviabilizando economicamente a produção e a qualidade da forragem exigida pelos animais. Tal processo pode ser de origem física, e/ ou química e/ou biológica. Neste trabalho, objetivou-se avaliar os efeitos do preparo do solo, correção da acidez e adubação em um Latossolo Amarelo distrocoeso de Tabuleiro Costeiro e as consequências no sistema radicular do B. decumbens. $O$ experimento foi localizado na área experimental da UFRB, Cruz das Almas - BA. O delineamento utilizado foi em parcelas subdivididas no espaço, contendo dois tratamentos de preparo do solo: gradagem pesada (Gp) e gradagem pesada + subsolagem (Gp+Subs) em três blocos e cinco combinações de fertilizantes e corretivos: Controle (Con); Calagem (Cal); NPK; Cal+NPK e Cal + Gesso Agrícola+NPK (CalGA+NPK). Foram avaliados: densidade, porosidade e resistência mecânica do solo à penetração; valores de $\mathrm{pH}, \mathrm{Al}^{3+}$ e V\%; e também densidade de raízes. Verificou-se que o efeito da subsolagem sobre os atributos físicos perdurou por dois anos, onde aumentou a porosidade do solo diminuiu a densidade e a resistência do solo à penetração radicular. A subsolagem interagiu com o calcário para correção do solo em profundidade.
\end{abstract}

Palavras-Chave: Resistência à penetração, Acidez do solo, Distribuição radicular

\section{Chemical and physical attributes of a yellow distrofic oxisol and root growth of Brachiaria decumbens submitted to fertilization and subsoiling}

\begin{abstract}
Pasture degradation is an evolutionary process of losing vigor, productivity and capacity of natural recovery of pastures. It can economically cripple the production and the forage quality required by the animals. This process can have a physical and/or chemical and/or biological origin. The aim of this study was to evaluate the effects of tillage, liming and fertilization on a yellow distrofic oxisol from a oastal land zone and its consequences on Brachiaria decumbens root system. The experiment was located at UFRB experimental area, Cruz das Almas - BA, Brazil. The experimental design was arranged in a split plot, with two tillage treatments: heavy disc harrowing (GP) and heavy disc harrowing + subsoiling (Gp + Subs) in three blocks and five fertilizers combinations: control (Test.); liming (Cal); NPK; NPK + Cal; and Cal + Agricultural Gypsum + NPK (CalGA + NPK). Were evaluated: density, porosity and the mechanical resistance of soil to penetration, $\mathrm{pH}, \mathrm{Al}^{3+}$ and $\mathrm{V} \%$ values and also root density. It was observed that the effect of subsoiling on soil physical attributes lasted for two years, increasing soil porosity and decreasing soil density and resistance to root penetration. Subsoiling interacted with lime leading to a depth correction.
\end{abstract}

Keywords: Penetration Resistance, Root distribution, Soil Acidity 


\section{Introdução}

A degradação de pastagens sobre solos tropicais altamente intemperizados está ligada ao declínio estrutural do solo e associado as perdas de matéria orgânica, o que afeta a dinâmica e a disponibilidade de nutrientes para as plantas. Essa degradação é controlada porum conjunto de fatores, incluindo as propriedades do solo, condições climáticas, características biológicas de espécies de gramíneas, manejo da pastagem, entre outros. (Bengough et al., 2011, Nesper et al., 2014).

A compactação, resultante da ação antrópica (transito de máquinas e equipamentos agrícolas, pisoteio de animais etc.) e o adensamento (consequência de processos naturais e pedogenéticos), tomam, muitas vezes, dimensões sérias pois são geralmente mencionadas como os fatores que influenciam diretamente a degradação das pastagens (Chen et al., 2014, Pringle et al., 2014). Segundo Rezende (2000), é a presença de horizontes subsuperficiais coesos, frequentes nos Latossolos dos Tabuleiros Costeiros, que dificultam a dinâmica da água no perfil e o seu armazenamento no solo, além de diminuir o volume de exploração do solo pelas raízes.

O preparo do solo (aração, gradagem, escarificação, subsolagem, etc.) visa à melhoria das condições físicas do leito de sementes e/ ou raízes, proporcionando-lhe benefícios na aeração, infiltração de água e disponibilidade de nutrientes para as plantas, além da redução da resistência do solo à penetração radicular, para que as plantas possam se desenvolver adequadamente (Rezende, 2013).

Nos Latossolos Coesos dos Tabuleiros Costeiros, o preparo convencional, com grade pesada, transporta para a camada superficial material da camada coesa subjacente, geralmente mais argilosa, mais ácida, pobre em nutrientes, com baixa CTC (Rezende et al., 2002). Assim, a subsolagem tem sido utilizada nesses solos para romper os horizontes adensados (Rezende, 2000; Nacif et al., 2008), objetivandose melhorar a movimentação de água no perfil, a disponibilidade de nutrientes e a penetração radicular ao longo do perfil do solo. De acordo com Lanças (2002), o princípio dessa operação é o rompimento do solo por propagação de trincas, mantendo a ordem natural de seus horizontes sem inverter a leiva, atuando nas suas linhas de fratura ou através das interfaces de seus agregados.

A maior parte da pesquisa agrícola em regiões tropicais tem-se centrado no desenvolvimento de métodos para identificar as necessidades de calagem para a correção do solo e na determinação das taxas e métodos de aplicação que resultem em maior resposta da cultura. Apesar de tais esforços, algumas abordagens foram desenvolvidas para determinar os processos e práticas de manejo de atividades que fazem com que ocorra o retorno de condições ácidas do solo. Assim, o objetivo deste trabalho foi avaliar os efeitos do preparo do solo, correção da acidez e adubação mineral sobre atributos físicos e químicos do solo e consequências no crescimento radicular de pastagem de B. decumbens em um Latossolo Amarelo Distrocoeso de tabuleiro Costeiro.

\section{Material e Métodos}

$O$ experimento foi instalado em uma área de pastagem de $B$. decumbens no campus da Universidade Federal do Recôncavo da Bahia (UFRB), no Município de Cruz das Almas, cuja precipitação pluviométrica média anual é de $1.170 \mathrm{~mm}$, variando entre 900 a $1.300 \mathrm{~mm}$, sendo os meses de março a agosto os mais chuvosos e setembro a fevereiro os meses mais secos. Os resultados da análise granulométrica e de fertilidade do solo na camada de 0,0-0,20 $\mathrm{m}$ foram o seguinte: $772,0 \mathrm{~g} \mathrm{~kg}-1$ de areia total, $90,81 \mathrm{~g} \mathrm{~kg}-1$ de silte e 137,19 g kg-1 de argila; $\mathrm{pH}$ $(\mathrm{H} 2 \mathrm{O})=5,05 ; \mathrm{Al}+3=0,15 \mathrm{cmolc} \mathrm{dm}-3 ; \mathrm{H}+\mathrm{Al}=3,0$ cmolc dm-3; $\mathrm{Ca}+2+\mathrm{Mg}+2=1,3$ cmolc dm-3; $\mathrm{K}+$ $=0,05 \mathrm{cmolc} \mathrm{dm}-3 ; \mathrm{CTC}-\mathrm{pH} 7=4,45 \mathrm{cmolc} \mathrm{dm}-3$; $\mathrm{P}=1,0 \mathrm{mg} \mathrm{dm}-3 ; \vee(\%)=32,5$ e $\mathrm{M} . \mathrm{O}=10,2 \mathrm{~g} \mathrm{dm}-3$. A área experimental foi de $8064 \mathrm{~m} 2$, dividida em três blocos de 174,0m x 6,0m, cujo delineamento experimental foi em parcelas subdivididas no espaço, contendo dois tratamentos de preparo do solo em três repetições e oito níveis de adubação mineral (subtratamentos) dentro de cada nível de preparo do solo cuja avaliação para a necessidade de corretivo e fertilizante, foi obtida na profundidade de 0,0-0,20 m. 
O preparo do solo foi realizado no ano de 2006, com gradagem pesada (Gp) e gradagem pesada + subsolagem (Gp+Subs). A gradagem foi realizada com grade pesada de 18 discos e a subsolagem foi realizada com subsolador de cinco hastes de 0,60 m de comprimento, com lâminas de corte sem asas, cuja profundidade operacional foi regulada para 0,45 m, conforme indicação da resistência à penetração (RP) antes do experimento. Após o preparo do solo, a área ficou em descanso durante dois anos para rebrota da gramínea. $\bigcirc$ calcário e o gesso agrícola foram aplicados no início de Março de 2008, a lanço na superfície do solo; a mistura fertilizante NPK, com a metade do N, foi aplicada no início de Junho de 2008; a segunda metade do $\mathrm{N}$ foi aplicada no início de Dezembro do mesmo ano. Com base nessas análises e no Manual de Adubação e Calagem do Estado da Bahia (1989).

Os tratamentos fertilizantes consistiram de controle (Cont); Calagem (Cal); NPK; Cal+NPK e Cal + Gesso Agrícola+NPK (CalGA+NPK). Foram aplicados $1200 \mathrm{~kg} \mathrm{ha}^{-1}$ de calcário (elevação de $\mathrm{V}$ para $50 \%$ ); $300 \mathrm{~kg} \mathrm{ha}^{-1}$ de gesso agrícola (correspondente à substituição de $25 \%$ do $\mathrm{CaO}$ do calcário); $80,0 \mathrm{~kg} \mathrm{ha}^{-1}$ de $\mathrm{N}$, na forma de Uréia, fracionada de duas vezes; $50,0 \mathrm{~kg} \mathrm{ha}^{-1}$ de $\mathrm{P}_{2} \mathrm{O}_{5^{\prime}}$ sob a forma de Superfosfato Triplo; $60 \mathrm{~kg}$ $\mathrm{ha}^{-1}$ de $\mathrm{K}_{2} \mathrm{O}$, sob a forma de $\mathrm{KCl}$.

Os efeitos dos tratamentos de preparo do solo sobre os atributos físicos foram avaliados por meio da densidade do solo (Ds), total de Poros (Pt), Macro (Mp) e Microporosidade (mp) e Resistência Mecânica do solo à penetração (RP). A Ds, Mp e mp foram determinados no início (Março de 2008) e no fim do período experimental (Agosto de 2009), utilizandose amostras indeformadas coletadas em três profundidades em cada bloco.

As avaliações da RP foram realizadas em dois períodos: agosto de 2008 - período chuvoso (80 mm); fevereiro de 2009 (10 mm) - período seco; e julho de 2009 (60 mm) - período chuvoso, utilizando-se penetrômetro de impacto modelo IAA - Planalsucar-Stolf, onde foram coletadas amostras nas profundidades de 0-0,20 m, 0,200,40 m e 0,40-0,60m para a determinação da umidade gravimétrica no momento da determinação da RP. Os efeitos dos tratamentos fertilizantes no solo foram avaliados por meio dos seguintes atributos: $\mathrm{pH}, \mathrm{Al}^{3+}$ e $\mathrm{V} \%$, avaliados nas camadas de 0-0,05 m, 0,05-0,20 m e 0,20$0,40 \mathrm{~m}$, em três épocas de avaliação: época 1 (Agosto de 2008 ou 5 meses após Calagem); época 2 (Fevereiro de 2009 ou 10 meses após a Calagem); e época 3 (Julho de 2009 ou 15 meses após a Calagem).

Para avaliação dos efeitos dos tratamentos sobre a pastagem, avaliou-se a matéria seca das raízes por volume de solo no perfil (densidade de massa seca de raízes DMR), cujo método de amostragem consistiu de prancha de pregos com 1,05 m x 0,60 m x 0,12 m, (Böhm, 1979), para retirada de monólitos de solo, na fase final do experimento. Assim, após a amostragem, os monólitos foram imersos em um tanque de água durante 48 horas e, em seguida, lavados, para retirada total do solo e exposição das raízes, as quais foram separadas nas camadas de 0-0,20 m; 0,20 m-0, 40 m; 0,400,60 m e 0,60-1,05 m e em seguida postas para secar em estufa de circulação forçada a 65 ${ }^{\circ} \mathrm{C}$, por 72 horas, para determinação da DMR. Os dados obtidos foram submetidos à análise de variância e teste de médias, utilizando o programa SISVAR (Ferreira, 2000).

\section{Resultados e Discussão}

\section{Atributos Químicos e Físicos}

A tabela 1 mostra os a avaliação dos diferentes atributos físicos do solo no início e final do experimento em função dos diferentes preparos adotados.

$\mathrm{Na}$ primeira avaliação, verifica-se que houve variação nos valores dos atributos físicos entre as camadas do solo submetidas ao mesmo preparo, com exceção de Pł e Ds no preparo Gp. Assim, os valores de Mp nas camadas 0,0-0,20 m e 0,20-0,40 m não diferiram entre si, mostrando, dessa maneira, o efeito da ação do subsolador na camada com presença de adensamento. Fato que é confirmado analisando o preparo $\mathrm{Gp}$, cujos valores diferiram nas três camadas.

Verifica-se ainda que o efeito do preparo do solo sobre os atributos, com exceção de Ds, na mesma profundidade só ocorreu na camada 
de 0,20-0,40 m, onde Gp+Subs proporcionou valor de Mp superior ao tratamento Gp. Assim, - método de preparo com Gp sobre esse atributo foi capaz de afetar apenas a camada mais superficial, pois, a Mp decresceu entre as camadas em enquanto que a adição da subsolagem na operação possibilitou os valores não significativos entre as primeiras camadas, pois, além de mobilizar a superfície do solo afetou também a camada subsuperficial $(0,20$ $0,40 \mathrm{~m})$.

Tabela 1. Atributos físicos do solo nas três profundidades avaliadas em função dos diferentes preparos do solo cultivado com $B$. decumbens.

\begin{tabular}{|c|c|c|c|c|c|c|c|c|}
\hline \multirow{2}{*}{ Prof. } & Gp+Subs & $\mathrm{Gp}$ & Gp+Subs & $\mathrm{Gp}$ & Gp+Subs & $\mathrm{Gp}$ & Gp+Subs & $\mathrm{Gp}$ \\
\hline & \multicolumn{2}{|c|}{$M p$} & \multicolumn{2}{|c|}{$\mathrm{mp}$} & \multicolumn{2}{|c|}{$\mathrm{Pt}$} & \multicolumn{2}{|c|}{ Ds } \\
\hline$m$ & \multicolumn{6}{|c|}{$\mathrm{m}^{3} \mathrm{~m}^{3}$} & \multirow{2}{*}{\multicolumn{2}{|c|}{$\left(\mathrm{kg} \mathrm{dm}^{-3}\right)$}} \\
\hline \multicolumn{7}{|c|}{ Início do Experimento } & & \\
\hline $0,00-0,20$ & $0,25 a A$ & $0,22 a A$ & $0,16 \mathrm{bA}$ & $0,17 \mathrm{bA}$ & $0,40 \mathrm{bA}$ & $0,38 a A$ & $1,55 \mathrm{aB}$ & $1,65 a \mathrm{~A}$ \\
\hline $0,20-0,40$ & $0,23 a A$ & $0,17 \mathrm{bB}$ & $0,20 \mathrm{bA}$ & $0,20 \mathrm{bA}$ & $0,43 a A$ & $0,36 \mathrm{aB}$ & $1,46 \mathrm{bB}$ & $1,60 \mathrm{aA}$ \\
\hline $0,40-0,60$ & $0,12 \mathrm{bA}$ & $0,12 \mathrm{CA}$ & $0,27 a A$ & $0,25 a A$ & $0,38 \mathrm{bA}$ & $0,37 a A$ & $1,66 \mathrm{aA}$ & $1,65 a \mathrm{~A}$ \\
\hline \multicolumn{9}{|c|}{ Final do Experimento } \\
\hline $0,00-0,20$ & $0,23 a A$ & $0,22 a A$ & $0,19 \mathrm{bA}$ & $0,19 \mathrm{bA}$ & $0,42 a A$ & $0,41 a \mathrm{~A}$ & $1,53 a A$ & $1,54 \mathrm{aA}$ \\
\hline $0,20-0,40$ & $0,18 \mathrm{bA}$ & $0,15 \mathrm{bA}$ & $0,22 a A$ & $0,23 a A$ & $0,40 \mathrm{aA}$ & $0,38 a A$ & $1,57 \mathrm{aA}$ & $1,63 a A$ \\
\hline $0,40-0,60$ & $0,08 c A$ & $0,10 c A$ & $0,24 a A$ & $0,23 a A$ & $0,33 \mathrm{bA}$ & $0,33 \mathrm{bA}$ & $1,66 \mathrm{aA}$ & $1,64 a \mathrm{~A}$ \\
\hline
\end{tabular}

Esse comportamento não foi verificado para as variáveis $\mathrm{mp}$ e Pt. Pois, no caso da $\mathrm{mp}$ não houve diferenças entre as camadas iniciais e nem entre o preparo do solo na mesma profundidade. Apenas o preparo com Gp+Subs influenciou na variável Pt, cujo valor em 0,20-0,40 m foi superior às demais camada. E, ainda, comparando o preparo do solo sobre essa variável na mesma profundidade, verificase que só houve efeito significativo na camada 0,20-0,40 m onde o preparo com Gp+Subs foi superior ao Gp. Oliveira et al., (2001) verificou que a mobilização superficial do solo com gradagem ocasionou redução na densidade e aumento da porosidade, Stone \& Silveira (2001) também verificou esse comportamento, porém, com compactação abaixo da profundidade de atuação do implemento.

A gradagem, cuja função principal é a desagregação do solo, ao mobilizar a camada mais superficial promove efeito imediato na porosidade e na densidade do solo cujo benefício logo desaparece, pois, as partículas de solo ao se reagruparem ocasionam rápida redução na macroporosidade do solo, isso é verificado na camada de 0,0-0,20 m em ambos os métodos, pois não há diferença significativa entre eles. No entanto as parcelas que foram submetidas à subsolagem apresentaram variação significativa na Mp e na Ds, camada intermediária $(0,20-0,40$ m) afetada pelas hastes do subsolador, cuja função é descompactar camadas adensadas em profundidade não alcançadas por outros implementos de preparo de solo (Grotta et al., 2004; Sazaki et al., 2005).

O efeito do preparo Gp+Subs sobre a Ds foi verificado na camada de 0,20-0,40 m pois, foi observado o menor valor e este diferiu dos encontrados nas camadas de 0,0-0,20 m e 0,40-0,60 m, apesar de não diferirem entre si. $\mathrm{E}$ para o preparo com Gp, não houve diferença entre os valores de Ds paras as camadas estudadas. Comparando o preparo nas mesmas profundidades, verifica-se na camada de 0,00,20 m e 0,20-0,40 m que os valores foram inferiores no preparo com Gp+Subs, demonstrando, assim, o efeito da subsolagem, também, nessa variável e que isso perdurou ao longo de dois anos após o preparo.

$\mathrm{Na}$ segunda avaliação, apesar de mantida as diferenças nas camadas no mesmo preparo, o efeito do preparo sobre essas variáveis não perdurou, pois não houve diferenças significativas sobre elas quando compara 0 preparo para a mesma camada do solo. Assim, a Mp e a mp comportou-se maneira semelhante, os valores diminuíram com a profundidade para ambos os métodos de reparo do solo. O mesmo ocorreu com Pł em relação ao preparo, no entanto os valores nas primeiras camadas não 
diferiram e para Ds não foi verificado diferenças entre as camadas e nem tampouco para o preparo. Esses comportamentos devem está relacionado com os rearranjos das partículas primárias e secundárias do solo após os três anos do preparo.

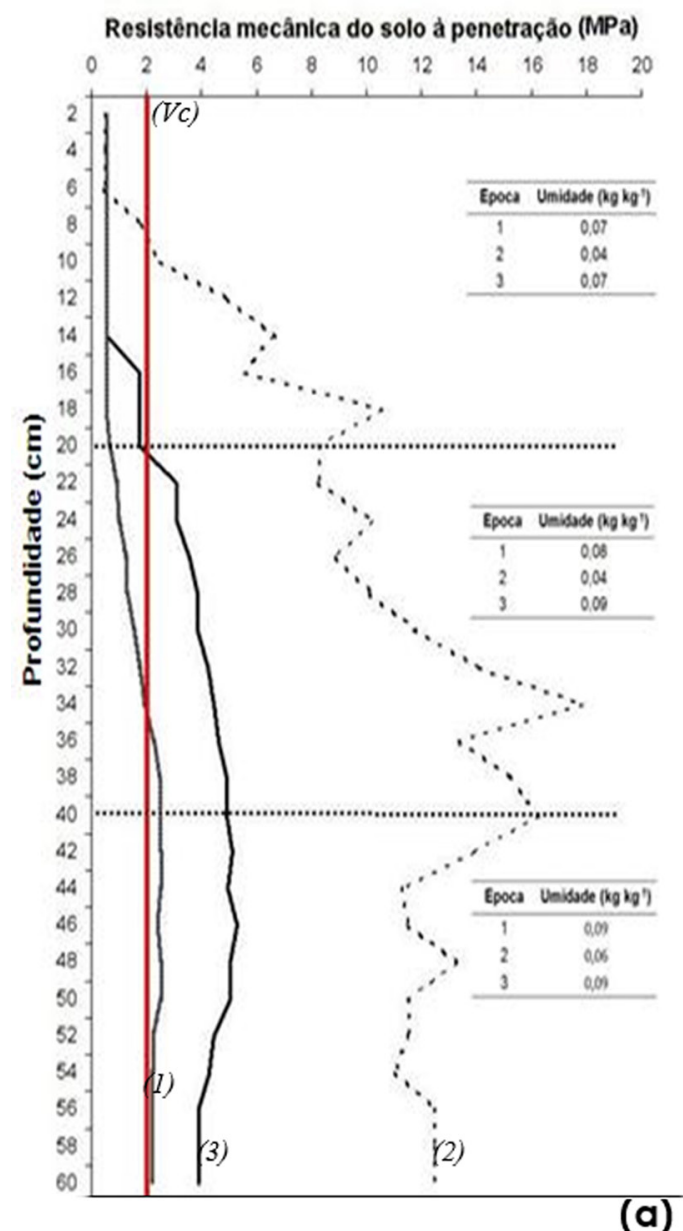

Para as mesmas condições de umidade, houve diferença significativa nos valores de resistência mecânica do solo à penetração devido aos métodos de preparo do solo (Figura 1).

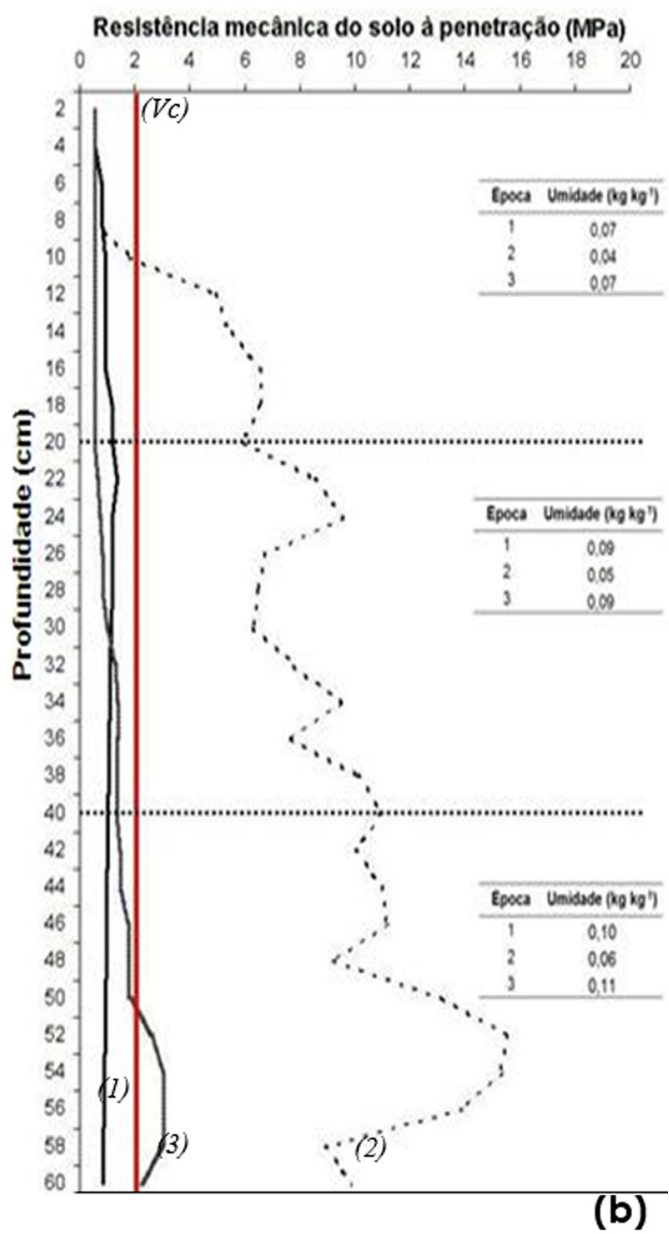

Figura 1. Resistência do solo à penetração (RP) em função do preparo do solo: (a) - Gradagem pesada e (b) Subsolagem+Gradagem pesada. 1, 2 e 3 são as épocas de avaliação. Vc - valor crítico de resistência à penetração adotada (2,0 MP).

No período chuvoso, nas camadas subsuperficiais preparadas com Gp+Subs, as hastes subsoladoras, proporcionaram valores de RP abaixo de 2,0 MPa, limite crítico ( $\mathrm{Vc}$ ) acima do qual, segundo Reichert et al. (2003), - desenvolvimento radicular da maioria dos vegetais cultivados é comprometido. Segundo esse autor, a resistência está estreitamente associada à densidade do solo e, para o mesmo teor de água, é tanto maior quanto maior a densidade, mostrando ser um bom indicador da compactação.
Para Rezende (2000), a aeração deficiente e a elevada resistência mecânica do solo à penetração, são as principais causas de inibição do crescimento radicular. O teste de média referente às interações entre preparo do solo e adubação mineral, nas três épocas de avaliação, é mostrado na tabela 2 e 3. Nota-se que a associação, Gp+Subs com a aplicação da calagem e/ou NPK, contribuiu para diferenças significativas entre as médias dos atributos químicos analisados em pelo menos uma das épocas. 
Tabela 2. Valores de saturação por bases do solo (V\%) em diferentes épocas de avaliação submetido a adubação e calagem em diferentes tipos de preparo do solo.

\begin{tabular}{|c|c|c|c|c|c|c|}
\hline \multirow{2}{*}{ Tratamentos } & \multicolumn{6}{|c|}{$\mathrm{V}(\%)$} \\
\hline & Gp+Subs & $\mathrm{Gp}$ & Gp+Subs & $\mathrm{Gp}$ & Gp+Subs & $\mathrm{Gp}$ \\
\hline & \multicolumn{6}{|c|}{ Primeira época de avaliação } \\
\hline & \multicolumn{6}{|c|}{ 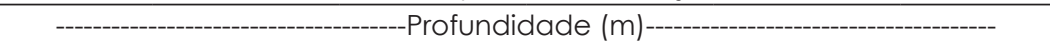 } \\
\hline & \multicolumn{2}{|c|}{$0,0-0,05$} & \multicolumn{2}{|c|}{$0,05-0,20$} & \multicolumn{2}{|c|}{$0,20-0,40$} \\
\hline Test & $57,50 \mathrm{aA}$ & $56,88 a A$ & $39,81 a$ & $34,53 \mathrm{aA}$ & $39,07 a A$ & $35,04 a \mathrm{~A}$ \\
\hline Cal & $69,83 a A$ & $69,70 \mathrm{aA}$ & $42,32 a$ & $45,88 \mathrm{aA}$ & $49,23 a A$ & $36,01 \mathrm{aB}$ \\
\hline NPK & $58,47 a A$ & $59,62 a A$ & $45,99 a$ & $38,45 a A$ & $35,98 a A$ & $30,36 a A$ \\
\hline Cal+NPK & $64,50 \mathrm{aA}$ & $63,14 \mathrm{aA}$ & $48,81 a \mathrm{~A}$ & $36,84 \mathrm{aB}$ & $39,63 a A$ & $32,85 a \mathrm{~A}$ \\
\hline \multirow[t]{2}{*}{ CalGA+NPK } & $58,53 a A$ & $50,98 \mathrm{aA}$ & $48,47 a$ & $39,07 \mathrm{aA}$ & $38,64 a \mathrm{~A}$ & $34,57 \mathrm{aA}$ \\
\hline & \multicolumn{6}{|c|}{ Segunda época de avaliação } \\
\hline Test & $40,82 \mathrm{bA}$ & $37,28 \mathrm{aB}$ & $29,77 \mathrm{bA}$ & $35,36 a \mathrm{~A}$ & $27,22 a A$ & $32,52 \mathrm{aA}$ \\
\hline Cal & $59,79 a A$ & $57,06 a A$ & $42,54 \mathrm{aA}$ & $45,34 \mathrm{aA}$ & $44,63 a \mathrm{~A}$ & $30,49 a \mathrm{~B}$ \\
\hline NPK & $43,07 \mathrm{bA}$ & $41,61 \mathrm{bA}$ & $32,37 \mathrm{bA}$ & $35,50 \mathrm{aA}$ & $28,14 \mathrm{aA}$ & $30,54 \mathrm{aA}$ \\
\hline Cal+NPK & $58,17 a A$ & $52,10 \mathrm{aA}$ & $34,27 \mathrm{bA}$ & $43,56 \mathrm{aA}$ & $27,81 a A$ & $32,19 a A$ \\
\hline \multirow[t]{2}{*}{ CalGA+NPK } & $55,74 a \mathrm{~A}$ & $45,81 \mathrm{bA}$ & $42,33 a A$ & $41,63 a A$ & $28,33 a A$ & $33,48 \mathrm{aA}$ \\
\hline & \multicolumn{6}{|c|}{ Terceira época de avaliação } \\
\hline Test & $52,81 \mathrm{bA}$ & $54,51 \mathrm{aA}$ & $39,04 a \mathrm{~A}$ & $37,00 \mathrm{aA}$ & $32,49 \mathrm{bA}$ & $35,04 \mathrm{bA}$ \\
\hline Cal & $72,55 \mathrm{aA}$ & $61,27 a A$ & $50,16 a A$ & $49,21 \mathrm{aA}$ & $44,09 \mathrm{aA}$ & $46,14 \mathrm{aA}$ \\
\hline NPK & $67,22 \mathrm{aA}$ & $58,14 \mathrm{aA}$ & $36,44 \mathrm{aA}$ & $44,92 \mathrm{aA}$ & $36,38 \mathrm{bA}$ & $33,60 \mathrm{bA}$ \\
\hline Cal+NPK & $64,08 \mathrm{aA}$ & $49,33 \mathrm{aB}$ & $42,98 \mathrm{aA}$ & $37,51 \mathrm{aA}$ & $39,26 \mathrm{aA}$ & $36,95 \mathrm{bA}$ \\
\hline CalGA+NPK & $63,47 a A$ & $52,04 a A$ & $43,61 a A$ & $39,75 a \mathrm{~A}$ & $47,13 a A$ & $41,79 \mathrm{aA}$ \\
\hline
\end{tabular}

Os valores de $\mathrm{pH}$ na camada mais superficial, apesar de não ocorrer diferença significativa, se manteve até a última avaliação acima de 5,0, principalmente naquelas parcelas submetidas à calagem. Assim, na primeira época de avaliação, não houve diferença significativa $(\operatorname{Pr}<0,05)$ nas camadas de 0,0-0,05 m e 0,20-0,40 $\mathrm{m}$ entre os tratamentos fertilizantes e o preparo do solo, no entanto, o preparo do solo com $\mathrm{Gp}+$ Subs nas parcelas contendo o tratamento Cal contribui para elevar os valores de $\mathrm{pH}$ na profundidade de 0,05-0,20 m. Nessa camada, comparando-se o método de preparo de solo nas parcelas submetido ao tratamento Cal, verifica-se que o valor de $\mathrm{pH}$ foi superior $(5,31)$ no Gp+Subs e relação ao Gp $(5,00)$.

A segunda época apresentou comportamento semelhante à época anterior, também não ocorreu diferença significativa $(\operatorname{Pr}<0,05)$ nas camadas de 0,0-0,05 $\mathrm{m}$ e 0,20$0,40 \mathrm{~m}$, ocorrendo diferenças na camada 0,05$0,20 \mathrm{~m}$, onde $\mathrm{o} \mathrm{pH}$ nas parcelas que receberam o tratamento Cal foi superior àqueles sem o corretivo e nas parcelas submetidas ao preparo Gp a aplicação de qualquer dos tratamentos fertilizantes não influenciaram nos valores desse atributo. Verifica-se que ocorreu influência do preparo do solo sobre o $\mathrm{pH}$, pois, no tratamento NPK, a parcela preparada com Gp houve diferença significativa no $\mathrm{pH}$ e este foi superior ao tratamento com Gp+Subs. E, na última época de avaliação, 15 meses após a calagem, os valores de $\mathrm{pH}$ não foram mais influenciados nem pelos tratamentos fertilizantes nem pelo método de preparo do solo, já que não mais diferem estatisticamente entre si.

De maneira geral, verifica-se redução dos valores de $\mathrm{Al}^{+}$até a última avaliação, ou seja, 15 meses após a calagem superficial.

Na primeira época, o preparo do solo não influenciou nos valores de $\mathrm{Al}^{+3}$ na camada de 0,0-0,05 m, no entanto houve efeito isolado do tipo de preparo do solo, pois os valores de $\mathrm{Al}^{+3}$, nas parcelas preparadas com Gp e contendo Cal, NPK e Cal + NPK, não diferiram entre si, porém, apresentaram valores inferiores ao tratamento testemunha e ao CalGA + NPK. Houve efeito isolado do preparo do solo na camada de 0,050,20 $\mathrm{m}$ nos tratamentos contendo Cal, cujos valores de $\mathrm{Al}^{+3}$ foram inferiores nas parcelas preparadas com Gp+Subs, comparado ao preparo Gp. Na camada de 0,20-0,40 m não houve interferência do método de preparo do solo, apenas os tratamentos Cal e/ou NPK 


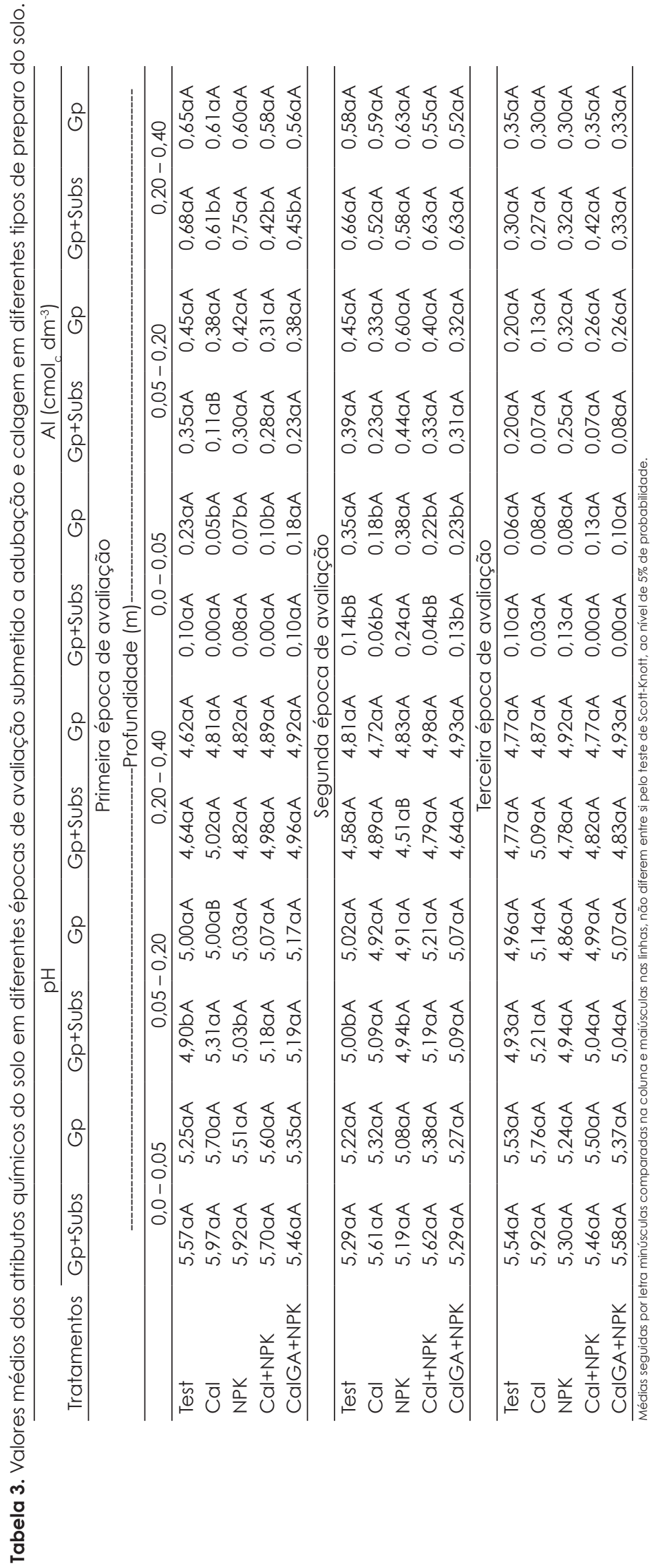

Com. Sci., Bom Jesus, v.6, n.4, p.385-395, Out./Dez. 2015 
influenciaram nos valores dessa variável, sendo que nas parcelas preparadas com Gp+Subs contendo Cal apresentaram menores valores de $\mathrm{Al}^{+3}$. Na segunda época apenas a camada de 0,0-0,05 m teve influência dos tratamentos. Observa-se que os valores de $\mathrm{Al}^{+3}$, nas parcelas submetidas ao preparo $\mathrm{Gp}+$ Subs com aplicação do tratamento Cal e o Cal+NPK foram inferiores ao tratamento submetidos ao preparo Gp. $\mathrm{E}$, na última época, os valores $\mathrm{Al}^{+3}$ não foram influenciados nem pelos tratamentos fertilizantes nem pelo método de preparo do solo.

Os valores de $\mathrm{Al}^{+3}$ na camada mais superficial ficam próximos à zero, principalmente nas parcelas onde a subsolagem foi combinada com a subsolagem, e nos tratamentos Cal e Cal+NPK $\circ \mathrm{Al}^{+3}$ foi totalmente neutralizado. Esse comportamento se repetiu na última época de avaliação nas parcelas submetidas ao tratamento Cal e no tratamento CalGA+NPK.

Verifica-se que, independente do método de preparo do solo, os valores de $\mathrm{V} \%$ na camada de 0,0-0,05 m ficaram sempre acima dos $50 \%$ nas parcelas submetidas à calagem em todas as épocas de avaliações. Na primeira avaliação na camada de 0,0-0,05 m, para os métodos de preparo do solo os tratamentos com adubação fertilizantes não influenciaram nos valores de $\mathrm{V} \% \mathrm{e}$, nas demais profundidades, o efeito do preparo ocorreu isoladamente, ou seja, para as parcelas submetidas ao tratamento Cal+NPK, camada 0,05-0,20 m, e, nas parcelas com Cal, 0,20-0,40 m o preparo do solo com Gp+Subs foi responsável pelo aumento da saturação por bases.

O mesmo foi observado na segunda época, na camada 0-0,05 m, parcela testemunha e nas parcelas contendo o tratamento Cal, camada 0,20-0,40 m, e, terceira época na profundidade na 0-0,05 m com o tratamento Cal+NPK. No entanto, a segunda época ocorreu efeitos isolados do preparo do solo sobre os valores de V\%. Assim, os menores valores na profundidade 0-0,05 $\mathrm{m}$ foram observados nos tratamentos sem calagem, nas parcelas submetidas ao Gp+Subs e no tratamento Cal+NPK submetida ao Gp. Na profundidade 0,05-0,20m nas parcelas submetidas ao Gp+Subs as parcelas com NPK e Cal+NPK foram superiores aos demais tratamentos e na última época de avaliação, camada de 0-0,05m, a testemunha foi inferior a todos os tratamentos no preparo Gp+Subs. As parcelas com NPK foram superiores e na camada 0,05-0,20 m não houve diferença significativa entre os tratamentos.

Nas parcelas contendo Cal, camada de 0,20-0,40 m, submetidas ao Gp+Subs os valores de $\mathrm{V} \%$ foram superiores aos demais e no preparo com Gp os tratamentos com Cal e Gp+Subs foram os que obtiveram os maiores valores de V\% e não houve diferença significativa entre si.

Entende-se que a variação nos valores dos atributos estudados foi causada pela movimentação de bases trocáveis no perfil do solo - facilitada pelo aumento da permeabilidade devido à combinação Gradagem Pesada e Subsolagem, e, conseqüentemente, o efeito corretivo na acidez do solo, se refletiu no aumento da saturação por bases.

Alguns pesquisadores (Alleoni et al., 2005; Pádua et al., 2006) têm mostrado que a reação do calcário aplicado a lanço fica restrita aos primeiros centímetros da camada superficial do solo devido à baixa solubilidade do carbonato de cálcio e ao menor contato de suas partículas com o meio. No entanto, em algumas situações, a reação da calagem superficial pode superar os $0,10 \mathrm{~m}$ de profundidade, uma vez que depende de fatores como: tipo de corretivo, dosagem, atributos físicos e químicos do solo e o regime hídrico de cada região (Caires et al., 2003; Fidalski \& Tormena, 2005).

\section{Densidade de massa seca de raiz}

A análise de variância mostrou efeito significativo (teste $F<0,05$ ) apenas para adubação mineral no crescimento radicular do capim B. decumbens. A figura 2 mostra os resultados do teste de média (Scott-Knott, $\mathrm{Pr}<$ $0,05)$ do desdobramento entre os tratamentos fertilizantes e o método de preparo para a DMR do capim. Na camada de $0,0-0,20 \mathrm{~m}$, as parcelas preparadas com Gp+Subs, todos os tratamentos foram superiores $(\operatorname{Pr}<0,05)$ ao controle e os maiores valores de DMR foram observados nos tratamentos Cal e no NPK, apesar de não diferirem entre si. 

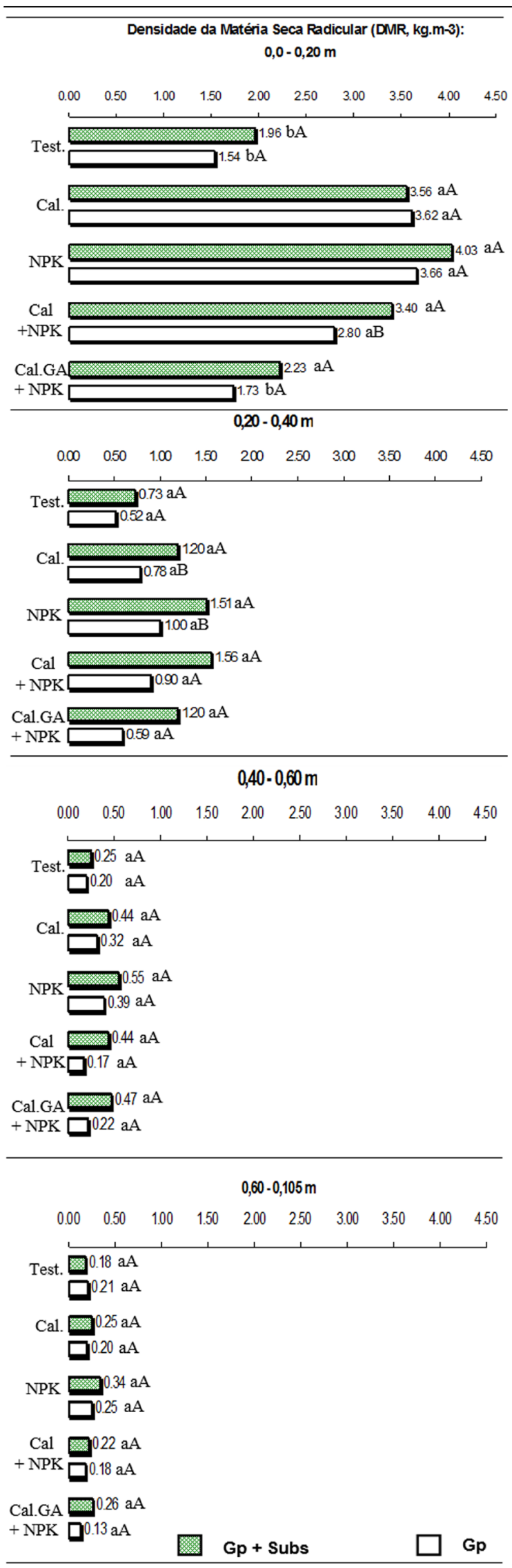

Figura 2: Densidade de matéria seca de raízes (DMS) ao longo do perfil do solo. Letras iguais minúsculas comparam - tratamento fertilizante nas camadas; as maiúsculas, o preparo do solo nas camadas. Quando iguais, não diferem estatisticamente entre si, pelo teste de Scott-Knott ao nível de $5 \%$ de probabilidade.
No preparo com Gp, os tratamentos controle e o CalGA+NKP não diferiram entre si e foram inferiores aos demais e semelhante ao preparo com Gp+Subs, os maiores valores de DMR foram verificados no Cal e no NPK. Assim, os valores de DMR, independente do preparo do solo indicam que a calagem e a aplicação da junção dos nutrientes NPK promoveram um aumento no crescimento radicular da gramínea. A contribuição do fósforo associado ao nitrogênio, potássio e/ou ao calcário para o ganho de massa de raiz se deve à melhoria da permeabilidade do solo à água promovida pelo preparo do solo. O fósforo é considerado nutriente de baixa mobilidade no solo. Assim, o preparo do solo com subsolagem aumentou a $\mathrm{Mp}$ - rota natural do crescimento radicular (KIEHL, 1979) e, conseqüentemente, o fluxo de água através do perfil, possibilitou maior mobilização desse nutriente por fluxo de massa e difusão, com reflexos positivos no crescimento radicular (Malavolta, 2006).

Processo semelhante pode ser relacionado ao cálcio do calcário: a melhoria do fluxo de água no solo favoreceu sua descida, contribuindo para a redução da acidez ativa $(\mathrm{pH})$ e trocável $\left(\mathrm{Al}^{+3}\right)$ e suprindo esse nutriente no ambiente radicular.

Em condições de equilíbrio desses macronutrientes na planta, há maior produção de fotoassimilados, que são redistribuídos para as raízes, aumentando o seu crescimento (Marschner, 1983). E ainda, uma planta bem suprida em $\mathrm{N}$ apresenta sistema radicular maior que uma planta deficiente em $\mathrm{N}$, porque os mecanismos envolvidos no crescimento do sistema radicular estão relacionados com o acúmulo de carboidratos (Brouwer, 1962).

Para a camada de 0,20-0,40 m, observase que os maiores valores de DMR são para as parcelas subsoladas, no entanto para o nível de significância $(\operatorname{Pr}<0,05)$ adotado não há diferenças significativa entre eles. O comportamento dos fertilizantes sobre os valores segue o mesmo comportamento, também não diferem entre si. Assim, verifica-se que os valores da variável em todos os tratamentos fertilizantes são superiores a testemunha e semelhante ao que ocorreu na camada mais superficial os maiores valores são 
verificados no tratamento contendo NPK e no Cal.

Os maiores valores de DMR apresentados nos tratamentos NPK e Cal em parcelas submetidas ao preparo Gp+Subs nessas camadas, estão relacionados à maior facilidade de crescimento sem restrições físicas como a resistência mecânica à penetração imposta pelo solo, ou ainda a uma maior aeração do sistema radicular promovidos pelo preparo em profundidade reduziu a resistência à penetração radicular permitindo o aumento na massa radicular.

Independente do método de preparo do solo e dos tratamentos fertilizantes, a DMR decresce com a profundidade e a maior concentração da massa de raízes ficou nas camadas mais superficial do solo, diminuindo consideravelmente a partir dos $0,60 \mathrm{~m}$ de profundidade. Doorenbos \& Pruitt (1977), relatando a absorção de água no perfil do solo, destacam que a maioria das plantas retira $40 \%$ do total de água utilizada do quarto superior do perfil do solo abrangido pelo sistema radicular e 30, 20 e 10\% da segunda, terceira e quarta frações do perfil do solo explorado pelas raízes, respectivamente. Para Bernardo et al. (2005), a profundidade efetiva do sistema radicular deve ser tal que, pelo menos, $80 \%$ das raízes das culturas estejam nela contida. E, de acordo com Zonta et al. (2006) a massa radicular (fresca ou seca) representa o estoque total de matéria subterrânea alocada, isto é, o conteúdo de reserva do vegetal e dessa forma, esse conteúdo refletirá em maior resistência do vegetal, períodos prolongados de déficit hídrico e maior capacidade de absorção de nutriente.

Para os dois métodos de preparo do solo, a densidade de raiz no tratamento com CalGA+NPK foi menor do que a observada nas parcelas adubadas com NPK e com NPK+Cal. Isso indica que os $300,0 \mathrm{~kg} \mathrm{ha}^{-1}$ de Gesso Agrícola combinados com o calcário não contribuíram para o desenvolvimento radicular. No entanto, encontra-se na literatura que o Gesso Agrícola é importante para o desenvolvimento radicular em profundidade e que além do suprimento de enxofre (Alvarez et al., 2001), sua aplicação junto com o calcário melhora a mobilização do cálcio ao longo do perfil do solo, facilitando a neutralização do efeito tóxico do $\mathrm{Al}^{3+}$. Isso contribui para melhorar o ambiente radicular das plantas (Caires et al., 2003).

\section{Conclusões}

O efeito da subsolagem sobre os atributos físicos avaliados na camada coesa permaneceu por dois anos, onde aumentou a $M p$, diminuiu a Ds e a resistência do solo à penetração radicular e contribuiu para maior movimentação dos nutrientes ao longo do perfil do solo.

\section{Referências}

Alleoni, L.R.F., Cambri, M.A., Caires, E.F. 2005. Atributos químicos de um latossolo de cerrado sob plantio direto, de acordo com doses e formas de aplicação de calcário. Revista Brasileira de Ciência do Solo 29: 923-934.

Bengough, A.G., Mckenzie, B.M., Hallett, P.D., Valentine, T.A. 2011 . Root elongation, water stress, and mechanical impedance: a review of limiting stresses and beneficial root tip traits. Journal of Experimental Botany 62: 59-68.

Bernardo, S., Soares, A.A., Mantovani, E.C. 2005. Manual de irrigação. 7.ed. Imprensa Universitária, Viçosa, Brasil. 611 p.

Böhm, W. 1979. Methods of studying root systems. Springer-Verlag, Berlim, Alemanhã. 185 p.

Brower, R. 1962. Nutritive influences on the distribution of dry matter in the plant. Nether. Journal Agricutural Science 10: 342-399.

Caires, E.F., Blum, J., Barth, G., Garbuio, F.J., Kusman, M.T. 2003 Alterações químicas do solo e resposta da soja ao calcário e gesso aplicados na implantação do sistema plantio direto. Revista Brasileira de Ciência do Solo 27(2): 275-286.

Chen, Y.L., Palta, J., Clements, J., Buirchell, B., Siddique, K.H., Rengel, Z. 2014. Root architecture alteration of narrow-leafed lupin and wheat in response to soil compaction. Field Crops Research 165: 61-70.

Doorenbos, J., Pruitt, J.O. 1977. Guidlines for predicting crop water requirements. FAO, Rome, Italia. $179 \mathrm{p}$.

Ferreira, D.F. 2000. Análises estatísticas por meio do Sisvar para Windows versão 4.0. In: Reunião anval da região brasileira da sociedade internacional de biometria. Anais... UFSCar: São Carlos, Brasil. p. 225-258.

Fidalski, J., Tormena, C.A. 2005. Dinâmica da 
calagem superficial em um Latossolo Vermelho distrófico. Revista Brasileira de Ciência do Solo 29: $235-247$

Grotta, D.C., Lopes, A., Furlani, C.E.A., Klinger, B.B., Reis, G.N.R., Silva, R.P. 2004. Subsolador: avaliação do desempenho em função da velocidade de trabalho e espaçamento entre hastes. Acta Scientiarum Agronomy 26(1): 21-26

Kiehl, E.J. 1979. Manual de Edafologia: relações solo-planta. Editora Agronômica CERES, São Paulo, Brasil. 262 p.

Lanças, P.K. 2002. Subsolagem ou Escarificação. Cultivar Máquinas 2(14): 38-42.

Malavolta, E. 2006. Manual de nutrição mineral de plantas. Ceres, São Paulo, Brasil. 638 p.

Marschner, H. General introduction to the mineral nutrition of plants. In: Lauchli, A.; Bieleski, R. L. (Ed.) 1983. Inorganic plant nutrition. Springer-Verlag, Berlin, Alemanha, v.15, p.5-60.

Nacif, P.G.S., Rezende, J.O., Fontes, L.E.F., Costa, L.M., Costa, O.V. 2008. Efeitos da subsolagem em propriedades físico-hídricas de um Latossolo Amarelo distrófico coeso do Estado da Bahia. Magistra 20: 186-192

Oliveira, J.O.A.P., Vidigal Filho, P.S., Tormena, C.A., Pequeno, M.G., Scapim, C.A., Muniz, A.S., Sagrilo, E. 2001. Influência de sistemas de preparo do solo na produtividade da mandioca. Revista Brasileira de Ciência do Solo 25: 443-450

Pádua, T.R.P., Silva, C.A., Melo, L.C.A. 2006. Calagem em Latossolo sob influência de coberturas vegetais: neutralização da acidez. Revista Brasileira de Ciência do Solo 30: 869-878

Reichert, J.M., Reinert, D.J., Braida, J.A. 2003. Manejo, qualidade do solo e sustentabilidade: condições físicas do solo agrícola. In: Congresso Brasileiro de Ciência do Solo, SBCS, Ribeirão Preto, Brasil. CD-ROM.

Rezende, J. O. 2000. Solos Coesos dos Tabuleiros Costeiros: limitações agrícolas e manejo. Série estudos agrícolas, 1. SEAGRI, Brasil, 117p.

Rezende, J.O., Magalhães, A.F.J., Shibata, R.T., Rocha, E.S., Fernandes, J.C., Brandão, F.J.C., Rezende, V.J.R.P. 2002. Citricultura Nos Solos Coesos Dos Tabuleiros Costeiros: análise e sugestões. SEAGRI/SPA, Salvador, Brasil. 97 p.

Rezende, J.O. 2013. Plantio direto de citros: mito ou realidade? Revista Bahia Agricola 4: 72-85

Nesper, M., Bünemann, E.K., Fonte, S.J., Rao, I.M., Velásquez, J.E., Ramírez,B., Oberson, A. 2014. Pasture degradation decreases organic $\mathrm{P}$ content of tropical soils due to soil structural decline. Geoderma. 257: 123-133.

Pringle, M.J., Allen, D.E., Phelps, D.G., Bray, S.G., Orton, T.G., Dalal, R.C. 2014. The effect of pasture utilization rate on stocks of soil organic carbon and total nitrogen in a semi-arid tropical grassland. Agriculture, Ecosystems \& Environment 195: 83-90.

Stone, L. F., Silveira, P. M. 2001 . Efeitos do sistema de preparo e da rotação de culturas na porosidade e densidade do solo. Revista Brasileira de Ciência do Solo 25(2): 395-401.

Sazaki, C.M., Gonçalves, J.L.M., Bentivenha, S.R.P. 2005. Desempenho operacional de hastes subsoladoras em função da ponteira e do tipo do solo. Scientia Forestalis 67: 44-52. 